Check for updates

Cite this: RSC Adv., 2017, 7, 32058

\section{Micro- and nano-patterns fabricated by embossed microscale stamp with trenched edges $\dagger$}

\begin{abstract}
Jin-Hyun Choi, Chang-Mok Oh and Jae-Won Jang (DD *
A submicron width of Au line patterns was fabricated from a polydimethylsiloxane (PDMS) line stamp of micron width by utilizing capillary-force and edge-transfer lithography (ETL). A template composed of $\mathrm{Al}(\mathrm{OH})_{3}$ was fabricated by capillary-force lithography (CFL) carried out by $\mathrm{KOH}$ ink-loaded microscale PDMS stamping on an $\mathrm{Au}$ nanomembrane $(30 \mathrm{~nm})$-deposited Al thin layer $(50 \mathrm{~nm})$ on a glass substrate. Because the $\mathrm{Au}$ nanomembrane works as an $\mathrm{OH}^{-}$ion channel, protruding $\mathrm{Al}(\mathrm{OH})_{3}$ micro-structures are obtained by a $\mathrm{pH}$ dependent molding process reacting underneath the $\mathrm{Al}$ thin layer. The protruding $\mathrm{Al}(\mathrm{OH})_{3}$ template has sharp edges at the molding area resulting from the capillary force working on the walls of the molding PDMS stamp and aqueous $\mathrm{Al}(\mathrm{OH})_{3}$ solution during the molding process. For example, the highest and widest ring edges of the protruding $\mathrm{Al}(\mathrm{OH})_{3}$ fabricated by a dot PDSM stamp ( $\phi: 4.5 \mu \mathrm{m}$, gap: $3 \mu \mathrm{m}$ ) are obtained through a molding time of 20 min with $1 \mathrm{M} \mathrm{KOH}$ ink. The protruding $\mathrm{Al}(\mathrm{OH})_{3}$ micro-structures were used as templates to fabricate dot and line PDMS stamps. The replicated PDMS stamps have deep trenches around the contact area of the stamps; thus, the replicated PDMS stamp has an advantage of holding ink solution in the recessed space. Through (ETL) with a replicated line PDMS stamp, which has deep trenches around the line shape contact area, an Au line-pattern array of $700 \mathrm{~nm}$ in width was fabricated by 16mercaptohexadecanoic acid (MHA) patterning on Au/Ti (30 nm/5 nm)-deposited silicon substrate and sequential Au etching for $20 \mathrm{~min}$. In this study, we successfully demonstrated that an advantageous PDMS stamp for (ETL) is obtained by a solid $\mathrm{Al}(\mathrm{OH})_{3}$ template fabricated by Au nanomembrane-based CFL.
\end{abstract}

Received 9th May 2017 Accepted 14th June 2017 DOI: $10.1039 / c 7 r a 05262 f$ rsc.li/rsc-advances stamped should be loaded in the more recessed parts than the protruding parts (contact area) of the PDMS stamp. Silane molecule inking on a microscale PDMS stamp by discontinuous dewetting, which has an effect on the selective inking of silane molecules at only recessed parts of the PDMS stamp, was carried out to generate down to $90 \mathrm{~nm}$-wide patterns. ${ }^{13} \mathrm{In}$ addition, sub-micron $(\sim 300 \mathrm{~nm})$ patterns of alkanethiols were reported by surface modification of the PDMS stamp to obtain selective inking on the PDMS stamp. The selective inking of molecules only on the recessed part of the PDMS stamp is very important in sub-micron patterning in ETL.

In this study, we develop a process to fabricate a PDMS stamp that is more suitable for conducting ETL; the PDMS stamp has deep trenches around its contact area to load more ink solution in its recessed part. In case of ETL with alkanethiol ink, additional treatment is required for the selective inking only on the recessed part of the PDMS stamp, due to absorption of alkanethiol inks into PDMS materials. ${ }^{14}$ Interestingly, the selective inking process is not necessary to carry out ETL with the PDMS stamp with trenches around the contact area. Moreover, as well as typical nanoscale contact printing, microscale printing is also available with the PDMS stamp with trenches. The PDMS stamp with trenches can transfer alkanethiol inks more densely at the edge than the contact area. Hence, two different alkanethiol self-assembled monolayers 
(SAMs) with different concentration can be generated. One is by typical microscale contact printing, the other is by ETL.

The PDMS stamp with deep trenches around the contact area was fabricated by a protruded $\mathrm{Al}(\mathrm{OH})_{3}$ template with sharp edges made from $\mathrm{Au}$ nanomembrane-based capillary-force lithography (CFL). In typical CFL, molding process by capillary-force working on recessed space of the stamp is a key phenomenon. ${ }^{15-17}$ Unlike most cases of CFL, inorganic materials can be molded with using Au nanomembrane as ion permeable channel. ${ }^{18}$ For example, protruded $\mathrm{Al}(\mathrm{OH})_{3}$ structures can be fabricated by a capillary-force assisting molding process conducted on an Au nanomembrane-deposited Al layer by a PDMS stamp. ${ }^{18}$ The edges of the protruded $\mathrm{Al}(\mathrm{OH})_{3}$ structures were tailored to be sharp by the capillary force working on the molded aluminum salt solution; thus, the sharp edges can be replicated as trenches when the PDMS stamp is fabricated by the protruded $\mathrm{Al}(\mathrm{OH})_{3}$ template. In the result, the sub-micron width of Au line patterns was obtained with ETL carried out with alkanethiol ink stamping by the replicated PDMS stamp on $\mathrm{Au}$ substrate and a sequential Au etching process. Originally, a line-shaped PDMS stamp (contact width: $10 \mu \mathrm{m}$, gap: $5 \mu \mathrm{m}$ ) was used to fabricate the protruded $\mathrm{Al}(\mathrm{OH})_{3}$ template by molding for $20 \mathrm{~min}$ with $1 \mathrm{M}$ aqueous $\mathrm{KOH}$ ink, with which the line PDMS stamp (contact width: $\sim 9 \mu \mathrm{m}$, gap: $\sim 6 \mu \mathrm{m}$ ) was replicated and used in ETL for the fabrication of $700 \mathrm{~nm}$-wide bi-lined Au patterns with $5 \mu \mathrm{m}$ intervals and $15 \mu \mathrm{m}$ of periodicity by stamping ethanolic 16-mercaptohexadecanoic acid (MHA) ink (10 mM) on an Au substrate (thickness: $30 \mathrm{~nm})$ and sequential Au etching for 20 min. We successfully demonstrated that $\mathrm{Au}$ nanostructures (700 nm-wide lines) can be obtained originally from the microscle PDMS stamp of $10 \mu \mathrm{m}$ in width. This methodology for fabricating a PDMS stamp with deep trenches around the contact area will be useful to conduct alkanethiol ink printing in ETL.

\section{Experiments}

\section{Fabrication of PDMS stamp using $\mathrm{Al}(\mathrm{OH})_{3}$ template by $\mathrm{Au}$ nanomembrane-based CFL}

As the first step, an Au nanomembrane (thickness: $30 \mathrm{~nm}$ )coated Al film (thickness: $50 \mathrm{~nm}$ ) on glass substrate was prepared by the thermal evaporation method (KVE-T2000, Korea Vacuum Tech., Korea). Two different dimensioned dotarray PDMS stamps (\#1. hexagonal close packing (hcp) lattice, $\phi: 4.5 \mu \mathrm{m}$, gap: $3 \mu \mathrm{m}$, depth: $700 \mathrm{~nm}, \# 2$. hcp lattice, $\phi: 4 \mu \mathrm{m}$, gap: $6 \mu \mathrm{m}$, depth: $700 \mathrm{~nm}$ ) and a line PDMS stamp (width: 10 $\mu \mathrm{m}$, gap: $5 \mu \mathrm{m}$, depth: $1 \mu \mathrm{m})$ with $1 \mathrm{M} \mathrm{KOH}$ ink solution $(0.4 \mu \mathrm{l})$ were used for positive-tone $\mathrm{Au}$ nanomembrane-based lithography. ${ }^{18}$ For fabrication of the dot-array and the line PDMS stamps, hole-array and line silicon templates with the opposite dimension to the PDMS stamp were fabricated by photolithography (Korea Advanced Nano Fab Center, KOREA) and sequential reactive ion etching (RIE) process (Korea Advanced Nano Fab Center, KOREA). The silicon templates were cleaned by oxygen plasma for $2 \mathrm{~min}$, and then overnight vapor coating of (heptadecafluoro-1,1,2,2-tetrahydrodecyl)trichlorosilane was carried out to make the surface of the $\mathrm{Al}(\mathrm{OH})_{3}$ templates hydrophobic. Resin and hardener for PDMS (Sylgard 184, Dow Corning, USA) with a mixture rate of $10: 1(\mathrm{w} / \mathrm{w})$ were poured on the $\mathrm{Al}(\mathrm{OH})_{3}$ templates, and the samples were baked in a convection oven (SH-VDO-30NH, SH Scientific, Korea) at $70{ }^{\circ} \mathrm{C}$ for 4 hours. ${ }^{19}$

To obtain edge-tailored protruded $\mathrm{Al}(\mathrm{OH})_{3}$ micro-structures, a relatively thin Al layer (thickness: $50 \mathrm{~nm}$ ), compared with typical positive-tone Au nanomembrane-based lithography, ${ }^{18}$ was used; non-sharp-edged protruded $\mathrm{Al}(\mathrm{OH})_{3}$ micro-structures were fabricated with a $100 \mathrm{~nm}$ Al layer underneath the $\mathrm{Au}$ nanomembrane. The positive-tone Au nanomembrane-based lithography was carried out with a molding time from 5 to $25 \mathrm{~min}$. After the positive-tone $\mathrm{Au}$ nanomembrane-based lithography, the fabricated $\mathrm{Al}(\mathrm{OH})_{3}$ micro-structures were gently washed by de-ionized (DI) water. The fabricated $\mathrm{Al}(\mathrm{OH})_{3}$ micro-structures were characterized with a scanning electron microscope (SEM) (JXA-8530F, JEOL, JAPAN) and an atomic force microscope (AFM) (Dimension Icon, Bruker Co., USA). The sharp-edged protruded $\mathrm{Al}(\mathrm{OH})_{3}$ micro-structures obtained with the dot-array and line PDMS stamps were used as templates to fabricate PDMS stamps. The replicated PDMS stamps were fabricated by aforementioned procedure of PDMS stamp fabrication with using the $\mathrm{Al}(\mathrm{OH})_{3}$ templates as masters.

\section{ETL for Au nano structure patterning using the PDMS stamp fabricated by CFL}

ETL with the line PDMS stamp with trenches around the contact area fabricated by CFL was carried out on a thermally evaporated $\mathrm{Au}$ film $(30 \mathrm{~nm})$ with a Ti buffer layer $(5 \mathrm{~nm})$ on silicon substrate with ethanolic MHA ink (10 mM). Excessively loaded MHA ink solution on the PDMS stamp was removed by preprinting several times on a sacrificial substrate in the printing process. Printed MHA-SAMs by the PDMS stamp worked as etching resist during the $\mathrm{Au}$ etching process carried out with aqueous $\mathrm{Au}$ etching solution containing $20 \mathrm{mM}$ thiourea, $30 \mathrm{mM}$ iron nitrate, $20 \mathrm{mM}$ hydrochloric acid, and $2 \mathrm{mM} 1-$ octanol. ${ }^{20-23}$ Rinsing with DI water and drying with $\mathrm{N}_{2}$ gas were carried out as the post-etching process of the samples. The Au nano/micro patterns fabricated by the ETL were characterized by an optical microscope (Axio Scope.A1, Carl Zeiss, Germany) and AFM (Dimension Icon, Bruker Co., USA).

\section{Results and discussion}

\section{CFL: fabrication of $\mathrm{Al}(\mathrm{OH})_{3}$ templates}

According to our previous report, ${ }^{18}$ Au nanomembrane (30 nmthick $\mathrm{Au}$ film) has pores of around $5 \mathrm{~nm}$ in diameter so that $\mathrm{OH}^{-}$ions can transfer through the Au nanomembrane. In the case of stamping $\mathrm{KOH}$ ink solution with a dot-array PDMS stamp on an $\mathrm{Au}$ nanomembrane-deposited $\mathrm{Al}$ layer, $\mathrm{Al}(\mathrm{OH})_{3}$ is molded in the space between the recessed part of the PDMS stamp and the Au nanomembrane (positive-tone Au nanomembrane nanolithography ${ }^{\mathbf{1 8}}$ ). In Fig. 1(a), details of the molding process are displayed. The Au nanomembrane is thermally deposited on a 50 nm-thick Al film-coated glass substrate (Fig. 1(a)I). A sufficient volume $(\sim 0.4 \mu \mathrm{l})$ of $1 \mathrm{M} \mathrm{KOH}$ 

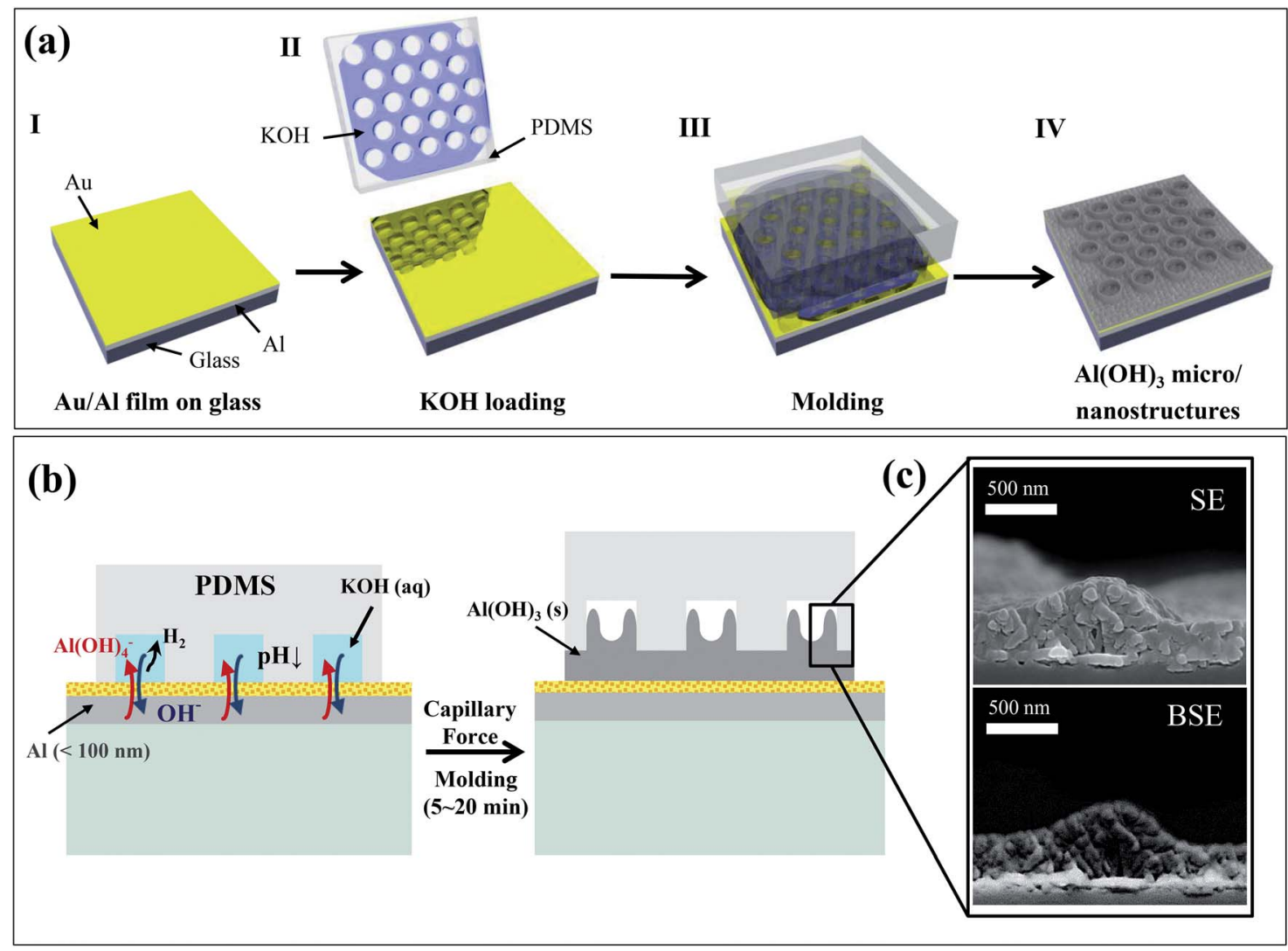

Fig. 1 (a) Scheme for fabrication of $\mathrm{Al}(\mathrm{OH})_{3}$ template by a molding process (positive-tone Au nanomembrane-based nanolithography). (b) Mechanism of the $\mathrm{Al}(\mathrm{OH})_{3}$ molding process carried out with CFL (molding with a relatively thin Al layer). (c) Cross-sectional SEM images (SE and BSE) of the $\mathrm{Al}(\mathrm{OH})_{3}$ template; an edge of the template has been sharpened.

ink is loaded on a PDMS stamp (i.e. dot-array stamp) to fully fill in recessed space of the PDMS stamp (Fig. 1(a)II). The KOH inkloaded PDMS stamp is placed on the Au nanomembrane/Al film-coated glass substrate for 5-25 min to mold aluminum salt $\left(\mathrm{Al}(\mathrm{OH})_{3}\right)$ in the recessed space of the PDMS stamp by utilizing the reaction between $\mathrm{Al}$ and $\mathrm{KOH} .{ }^{18}$ (Fig. 1(a)III and 1(b)) As shown in Fig. 1(b), the main mechanism of the $\mathrm{Al}(\mathrm{OH})_{3}$ molding process is that as follows: the liquid phase of aluminum salt $\left(\mathrm{Al}(\mathrm{OH})_{4}{ }^{-}\right.$(aq)) is formed by a reaction between the $\mathrm{Al}$ layer underneath the $\mathrm{Au}$ nanomembrane and $\mathrm{OH}^{-}$ions that have penetrated through the Au nanomembrane (eqn (1)), and the solid phase of aluminum salt $\left(\mathrm{Al}(\mathrm{OH})_{3}(\mathrm{~s})\right)$ is obtained as the $\mathrm{pH}$ decreases in the liquid phase of aluminum salt ${ }^{18}$ (eqn (2)). Because the volume of the molding space (the volume of the recessed space of the PDMS stamp) is not sufficiently large, the $\mathrm{pH}$ decreases by consuming $\mathrm{OH}^{-}$ions in the reacting solution of the molding space. ${ }^{18}$ Finally, protruded $\mathrm{Al}(\mathrm{OH})_{3}$ patterns with opposite dimensions to the PDMS stamp can be obtained on the Au nanomembrane, as shown in Fig. 1(a)IV. Chemical reactions related to the molding process of $\mathrm{Al}(\mathrm{OH})_{3}$ are shown in eqn (1) and (2). ${ }^{24,25}$

$$
\begin{gathered}
2 \mathrm{Al}(\mathrm{s})+6 \mathrm{H}_{2} \mathrm{O}(\mathrm{l})+2 \mathrm{OH}^{-}(\mathrm{aq}) \rightarrow 2 \mathrm{Al}(\mathrm{OH})_{4}{ }^{-}(\mathrm{aq})+3 \mathrm{H}_{2}(\mathrm{~g}) \\
2 \mathrm{Al}(\mathrm{OH})_{4}{ }^{-}(\mathrm{aq}) \rightarrow 2 \mathrm{Al}(\mathrm{OH})_{3}(\mathrm{~s})+\mathrm{OH}^{-}(\mathrm{aq})
\end{gathered}
$$

The edges of the molded $\mathrm{Al}(\mathrm{OH})_{3}$ structures, which are protruded and have opposite dimensions to the PDMS stamp used in the molding process, can be tailored by using a relatively thin Al layer underneath the Au nanomembrane. In the case of CFL carried out on polystylene (PS) polymer spin-coated substrate with a PDMS stamp that is several micrometers $(\sim 2.5$ $\mu \mathrm{m})$ deep, a relatively thin PS layer (thickness: $\sim 50 \mathrm{~nm}$ ) has been used to form the molded PS structures with higher edges as a result of CFL. ${ }^{26}$ In our study, a relatively thin Al layer $(<100 \mathrm{~nm})$ compared with the depth of the PDMS stamp $(700 \mathrm{~nm})$ was also used to ensure the sharp edges of the molded $\mathrm{Al}(\mathrm{OH})_{3}$ micro-structures (Fig. 1(b)). If a dot-array PDMS stamp is used in the molding process with the thin Al layer underneath the Au nanomembrane, due to the sharp edges of the molded $\mathrm{Al}(\mathrm{OH})_{3}$ structures in the recessed space of the dot-array PDMS stamp, a ring-like $\mathrm{Al}(\mathrm{OH})_{3}$ structure array can be obtained, as shown in Fig. 1(a)IV. Fig. 1(c), cross-sectional SEM images of the ring-like $\mathrm{Al}(\mathrm{OH})_{3}$ structure, clearly shows that the edges of the molded $\mathrm{Al}(\mathrm{OH})_{3}$ micro-structures are tailored to be sharp. In addition, an approximately $200 \mathrm{~nm}$-thick $\mathrm{Al}(\mathrm{OH})_{3}$ layer is also formed above the $\mathrm{Au}$ nanomembrane, and the $\mathrm{Au}$ nanomembrane is represented with high contrast in the backscattered electron (BSE) mode SEM image (Fig. 1(c)). If KOH ink solution is not entirely removed from the contact of the PDMS stamp, aluminum salts could be solidified in the gap between the contact area of the PDMS stamp and the Au 
nanomembrane; thus, a thin $\mathrm{Al}(\mathrm{OH})_{3}$ layer would form above the $\mathrm{Au}$ nanomembrane, as shown in Fig. 1(b) and (c). The $\mathrm{Al}(\mathrm{OH})_{3}$ micro-structures with tailored edges fabricated by the molding process carried out with CFL can be used as templates to fabricate a PDMS stamp with specific dimensions derived from the tailored $\mathrm{Al}(\mathrm{OH})_{3}$ micro-structures.

The molding time dependence of $\mathrm{Al}(\mathrm{OH})_{3}$ micro-structures by CFL with a dot-array PDMS stamp (hcp lattice, $\phi: 4.5 \mu \mathrm{m}$, gap: $3 \mu \mathrm{m}$ ) is characterized in Fig. 2 . To carefully characterize the dimensions of the molded ring-like $\mathrm{Al}(\mathrm{OH})_{3}$ microstructures, the heights at the edge and center of the protruded ring-like $\mathrm{Al}(\mathrm{OH})_{3}$ micro-structures, respectively denoted as $h_{\text {edge }}$ and $h_{\text {center }}$, as shown in Fig. 2(a), are measured by increasing the molding time from $5 \mathrm{~min}$ to $25 \mathrm{~min}$. Fig. 2(a) shows a scheme of the cross-sectional dimensions of the molded ring-like $\mathrm{Al}(\mathrm{OH})_{3}$ micro-structures and how to determine $h_{\text {edge }}$ and $h_{\text {center }}$ of the $\mathrm{Al}(\mathrm{OH})_{3}$ micro-structures. The molding time-dependent $h_{\text {edge }}$ and $h_{\text {center }}$ are displayed in Fig. 2(b). Until $15 \mathrm{~min}, h_{\text {edge }}$ and $h_{\text {center }}$ slightly increase to $50 \mathrm{~nm}$ with increases of the molding time. The $h_{\text {edge }}$ remarkably increases to $335 \mathrm{~nm}$ at $20 \mathrm{~min}$ of molding time and does not increase more (it seems to be saturated), whereas $h_{\text {center }}$ remarkably and gradually increases at the molding times of $20 \mathrm{~min}$ and $25 \mathrm{~min}$. Because a ring-like shape can be observed at the edges of the protruded $\mathrm{Al}(\mathrm{OH})_{3}$ micro-structures, the dimensions of the ring, such as height (ring height) and width (ring width), are also measured as the molding time increases (Fig. 2(c)). In Fig. 2(d), it turns out that relatively high and thick ring structures are obviously measured in the molded $\mathrm{Al}(\mathrm{OH})_{3}$ micro-structures; topographic AFM images of the molding time-dependent $\mathrm{Al}(\mathrm{OH})_{3}$ micro-structures clearly show that obvious ring structures at the edges of the protruded $\mathrm{Al}(\mathrm{OH})_{3}$ micro-structures are fabricated in $20 \mathrm{~min}$. The schematic images at the right side of Fig. 2(d) represent the cross-sectional structures of the molded $\mathrm{Al}(\mathrm{OH})_{3}$ at every molding time. Fig. 2(c) shows that the ring height and ring width decrease at the molding time of $25 \mathrm{~min}$, which can be regarded as the $\mathrm{Al}(\mathrm{OH})_{3}$ starting to solidify near the walls of the PDMS stamp, then continuing to become solidified at the center of the recessed space of the PDMS stamp. The molding time-dependent cross-sectional schematic images of the $\mathrm{Al}(\mathrm{OH})_{3}$ structures are represented at the right side of Fig. 2(d). The edge of the molded $\mathrm{Al}(\mathrm{OH})_{3}$ micro-structures with (a)

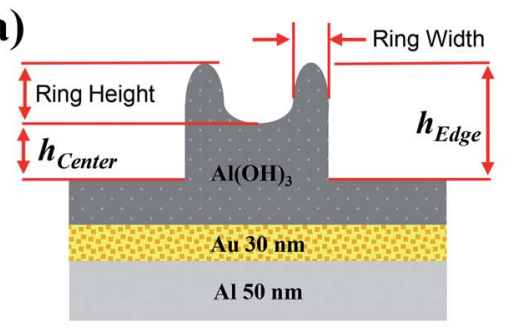

(b)

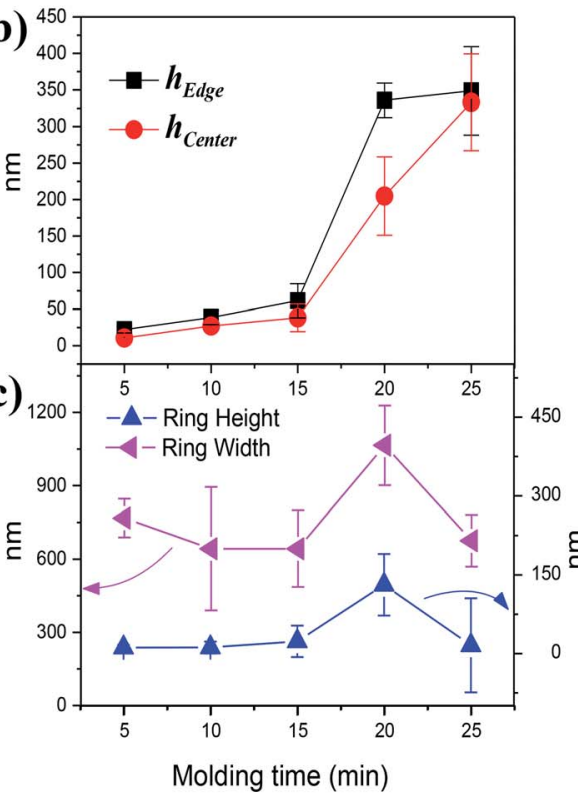

(d)
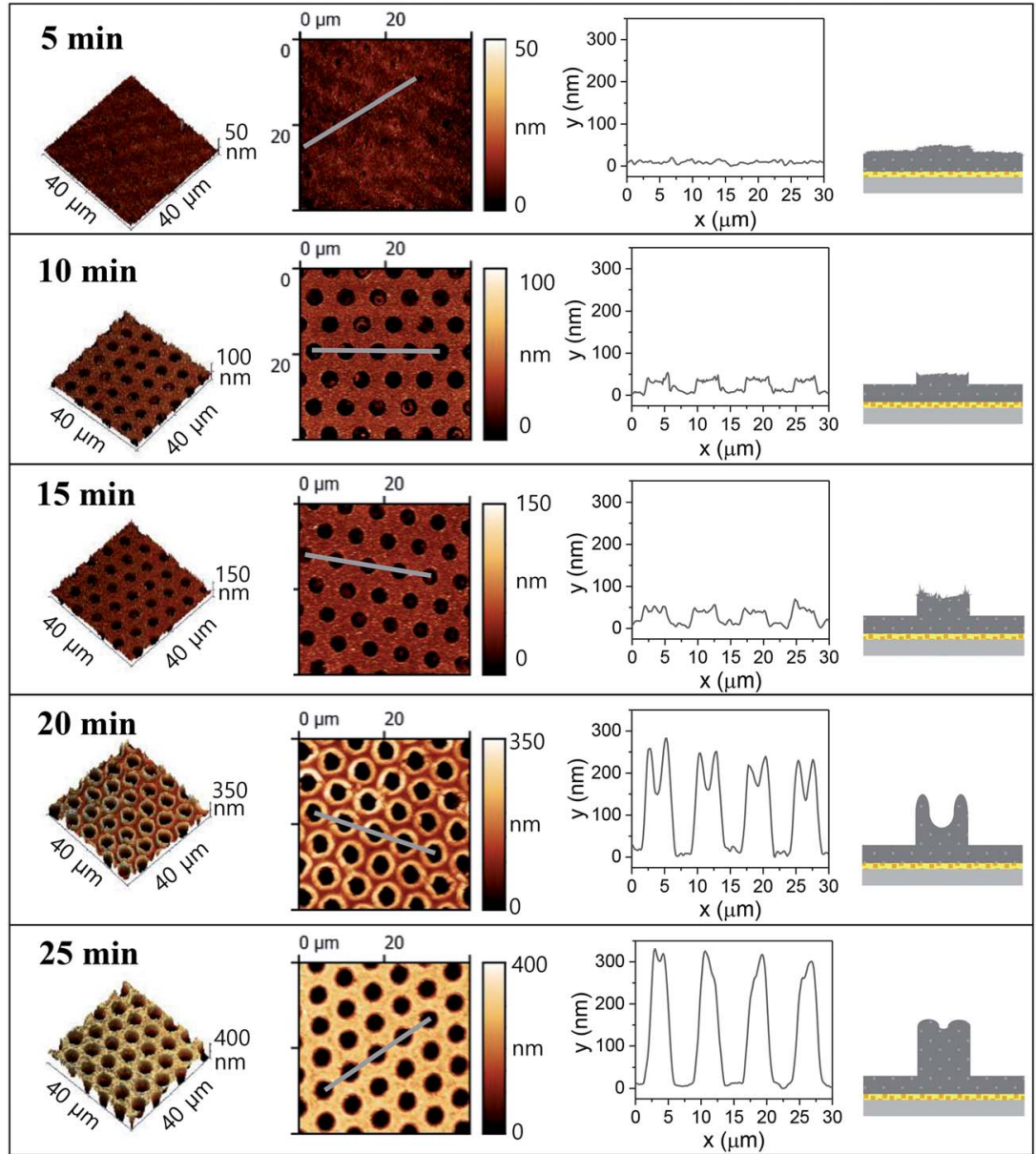

Fig. 2 (a) Scheme of the $\mathrm{Al}(\mathrm{OH})_{3}$ micro-structures fabricated by the molding process. Parameters for dimensions of the $\mathrm{Al}(\mathrm{OH})_{3}$ microstructures ( $h_{\text {edge, }} h_{\text {center, }}$ ring height, and ring width) are determined as displayed. (b, c) Graphs of molding time-dependent dimensional parameters of the $\mathrm{Al}(\mathrm{OH})_{3}$ micro-structures. (d) 3-Dimensional (3-D) and 2-dimensional (2-D) topological AFM images with cross-sectional line profile (obtained from the grey lines in 2-D topological AFM images) and scheme of the molding time dependent $\mathrm{Al}(\mathrm{OH})_{3} \mathrm{micro}-\mathrm{structures}$. 
a PDMS stamp can be tailored by properly adjusting the molding time; thus, the ring-like structure array of $\mathrm{Al}(\mathrm{OH})_{3}$ is obtained by 20 min of molding time with the dot-array PDMS stamp.

ETL: nano-patterning by the PDMS stamp obtained from the $\mathrm{Al}(\mathrm{OH})_{3}$ template

The edge-tailored $\mathrm{Al}(\mathrm{OH})_{3}$ micro-structure array can be used as a template for fabricating a PDMS stamp that is suitable for ETL. The sharp edge of the $\mathrm{Al}(\mathrm{OH})_{3}$ micro-structure can be transferred as a trench around the contact area of the replicated PDMS stamp, because a fabricated PDMS stamp by the $\mathrm{Al}(\mathrm{OH})_{3}$ micro-structure template has opposite dimensions. In Fig. 3(a), a scheme of fabrication of the replicated PDMS stamp from an $\mathrm{Al}(\mathrm{OH})_{3}$ template with a ring-like structure array is displayed. A ring-like $\mathrm{Al}(\mathrm{OH})_{3}$ micro-structure array fabricated by a hcp lattice dot-array PDMS with a $4 \mu \mathrm{m}$ diameter and $6 \mu \mathrm{m}$ gap is shown in Fig. 3(b), which was fabricated by 20 min of molding time with $1 \mathrm{M} \mathrm{KOH}$ ink solution. The fabricated ring-like $\mathrm{Al}(\mathrm{OH})_{3}$ micro-structure template shows that $h_{\text {edge }}, h_{\text {center }}$, ring height, and ring width are $0.32 \mu \mathrm{m}, 0.21 \mu \mathrm{m}, 0.13 \mu \mathrm{m}$, and 1.06 $\mu \mathrm{m}$, respectively (Fig. 3(b)). As shown in Fig. 3(c), a dot-array PDMS stamp with a trench around its contact area was replicated by the $\mathrm{Al}(\mathrm{OH})_{3}$ micro-structure template. According to the AFM topographic image of the replicated PDMS stamp (Fig. 3(c)), the trenches of the replicated PDMS stamp show
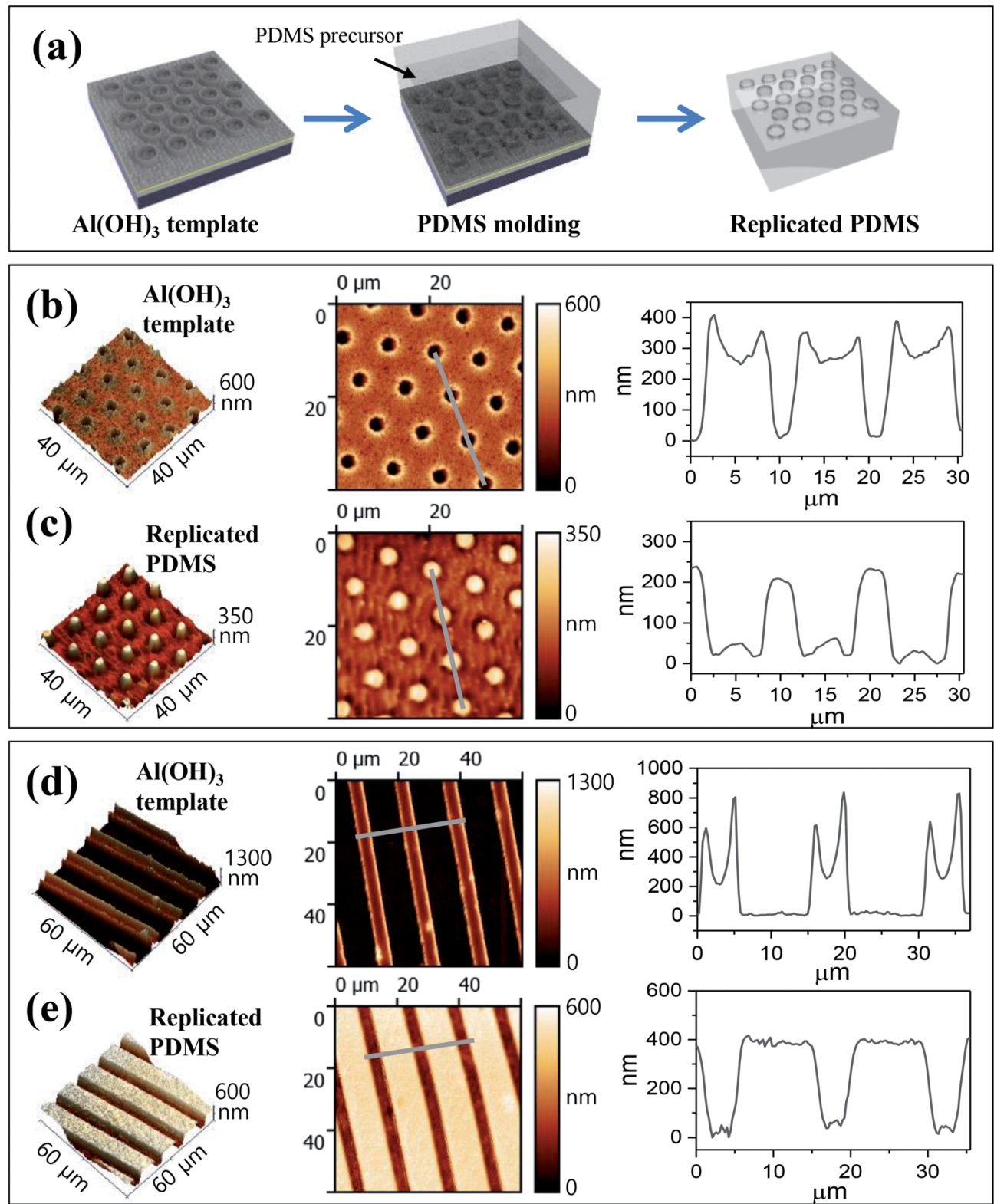

Fig. 3 (a) Scheme of PDMS stamp replication from the $\mathrm{Al}(\mathrm{OH})_{3}$ template fabricated by CFL. (b-e) 3-D and 2-D topological AFM images and crosssectional line profile: (b) a ring-like $\mathrm{Al}(\mathrm{OH})_{3}$ micro-structure array fabricated by a hcp lattice dot-array PDMS ( $\left.\phi: 4 \mu \mathrm{m}, \mathrm{gap}: 6 \mu \mathrm{m}\right)$, (c) the replicated PDMS stamp by (b), (d) an edge-tailored line shape $\mathrm{Al}(\mathrm{OH})_{3}$ micro-structure template fabricated by a line-shaped PDMS stamp (width: $10 \mu \mathrm{m}$, gap: 5 $\mu \mathrm{m})$, and (e) the replicated PDMS stamp by (d). The cross-sectional line profiles were measured from the grey lines in the 2-D topological AFM images. 
opposite dimensions to the ring shape of the $\mathrm{Al}(\mathrm{OH})_{3}$ template; the depth is $40 \mathrm{~nm}$, and the width is $1.9 \mu \mathrm{m}$.

As well as the ring-like array $\mathrm{Al}(\mathrm{OH})_{3}$ micro-structure template (Fig. 3(b)), an $\mathrm{Al}(\mathrm{OH})_{3}$ micro-structure template fabricated by a line-shaped PDMS stamp (width: $10 \mu \mathrm{m}$, gap: $5 \mu \mathrm{m}$ ) was obtained (Fig. 3(d)) and used for fabricating the replicated PDMS stamp, as shown in Fig. 3(c) and (e). Similar to the replicated PDMS stamp with the ring-like $\mathrm{Al}(\mathrm{OH})_{3}$ micro-structure template, the sharp edges of the $\mathrm{Al}(\mathrm{OH})_{3}$ micro line array structured template (Fig. 3(d)) are transferred as trenches in the replicated line PDMS stamp (Fig. 3(e)). The replicated PDMS stamps have relatively smaller height of protruding part (contact area) than depth of the opposite dimensioned recessed space in the $\mathrm{Al}(\mathrm{OH})_{3}$ templates, which can be assumed to be due to the fact that the PDMS materials were not fully filled into the recessed space of the $\mathrm{Al}(\mathrm{OH})_{3}$ templates during the replication (Fig. S5 $\dagger$ ).

Trenches around the contact area of the ring and the lineshaped replicated PDMS stamp can work as reservoirs to hold more volume of an ink solution in the recessed space of the
PDMS stamp; thus, the improved performance of the replicated PDMS stamp shown in Fig. 3(c) and (e) is expected when it is used in ETL. ETL carried out by the line-shaped replicated PDMS stamp is demonstrated in Fig. 4. MHA ink is used to pattern an Au film (30 nm) coated on glass substrate with a thin Ti buffer layer ( $5 \mathrm{~nm}$ ), as shown in Fig. 4(a)I. During the printing process, MHA ink will be loaded more in the recessed space by the trenches around the contact areas, as shown in the schematic images of Fig. 4(a)I. Due to the larger volume of MHA ink on the trenches of the PDMS stamp than the contact area of the PDMS stamp, the more condensed MHA-SAMs will be patterned at the edges of the PDMS stamp rather than the center of the contact area of the PDMS stamp, as shown in the schematic images of Fig. 4(a)I and II. By means of two different densities of MHA-SAM patterns at the edge and center of the PDMS stamped area, the Au film will be sequentially etched in the following order: non-patterned area, the center of the MHA-SAM patterns, and the edge of the MHA-SAM patterns. Fig. 4(a)III and (b) show that only the Au film in the non-patterned area is removed for

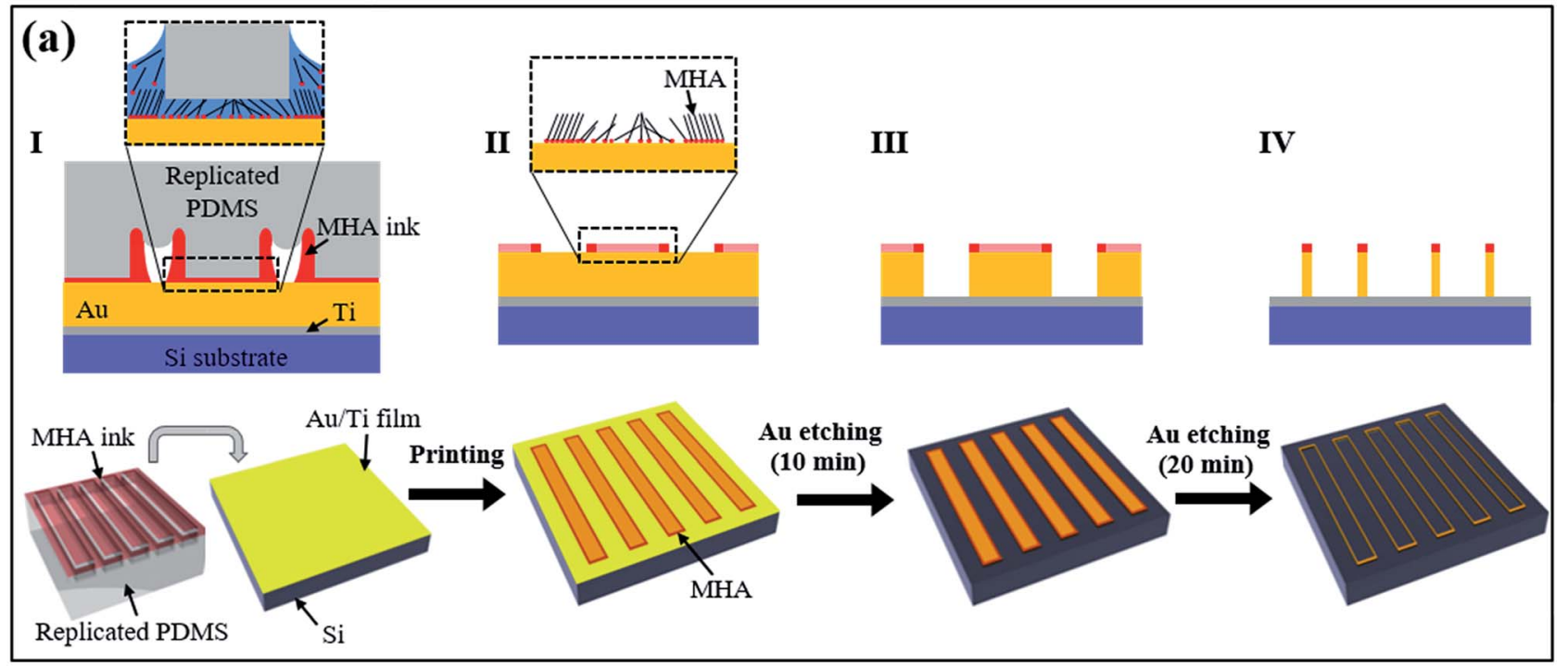

\section{(b)}

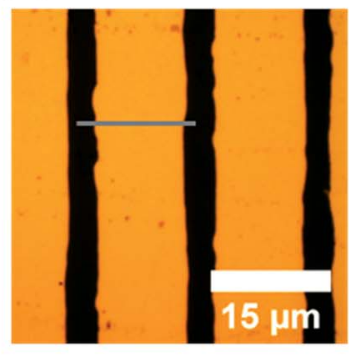

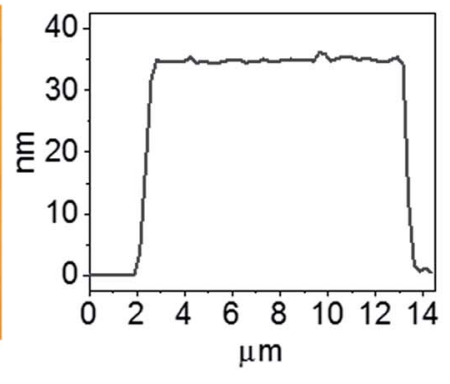

(c)
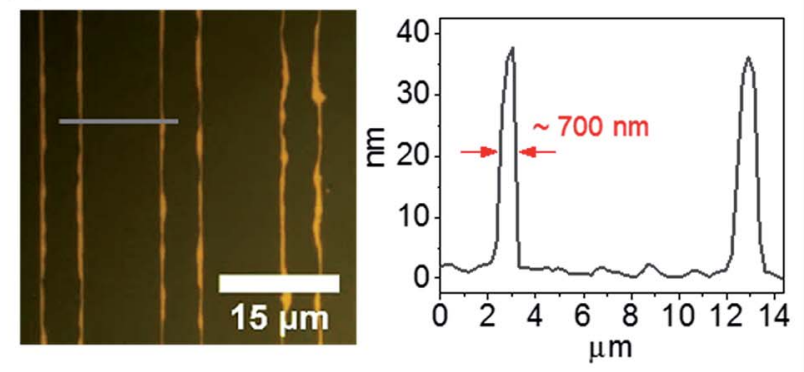

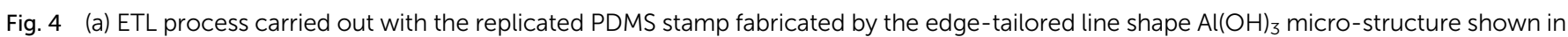

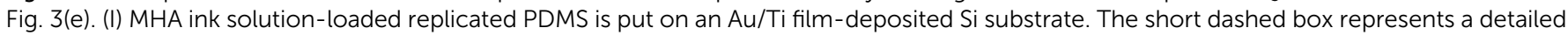

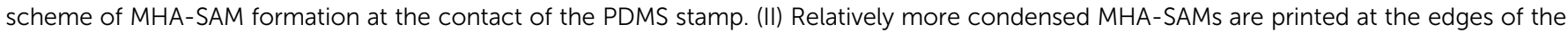

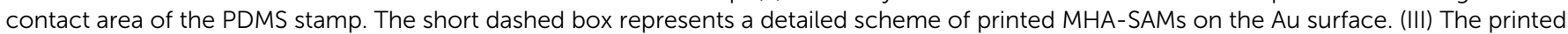

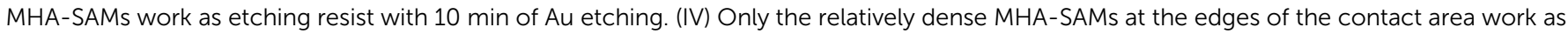

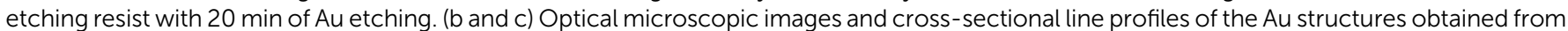

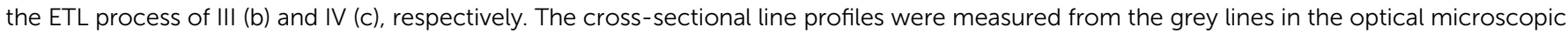
images. 
10 min of Au etching time. When the etching time increases up to 20 min (Fig. 4(a)IV), the Au film underneath the center of the MHA-SAM patterns is also etched away; thus, the sub-micron width $(\sim 700 \mathrm{~nm})$ of the Au bi-lines is obtained, as shown in Fig. 4(c). The cross-sectional line profile shown in Fig. 4(b) represents that approximately $11 \mu \mathrm{m}$-wide and $35 \mathrm{~nm}$-thick line patterns were obtained by 10 min of Au etching. Considering that the replicated line PDMS stamp (Fig. 3(e)) has approximately $9 \mu \mathrm{m}$-wide contact line patterns with an approximately 6 $\mu \mathrm{m}$ gap, it can be confirmed that MHA-SAMs were printed up to $1 \mu \mathrm{m}$ away from the edges of the contact area of the line PDMS stamp. In addition, the MHA-SAMs printed at the outside edges of the line PDMS stamp work more efficiently as resist during the etching process until $20 \mathrm{~min}$; the $35 \mathrm{~nm}$-thick and approximately $700 \mathrm{~nm}$-wide patterns were measured at the girth of the $11 \mu \mathrm{m}$-wide Au pattern, as is clearly shown in Fig. 4(c).

\section{Conclusions}

In conclusion, we have demonstrated that $\mathrm{Au}$ nanoscale line structures can be fabricated by the ETL process using a microscale line-shaped PDMS stamp replicated by an edge-tailored $\mathrm{Al}(\mathrm{OH})_{3}$ micro-structured template obtained by an $\mathrm{Au}$ nanomembrane-based CFL molding process (positive-tone nanolithography). In this study, a relatively thin Al layer $(<100$ $\mathrm{nm}$ ) was introduced in Au nanomembrane-based positive-tone lithography ${ }^{\mathbf{1 8}}$ for the molding of aluminum salt $\left(\mathrm{Al}(\mathrm{OH})_{3}\right)$ with sharply tailored edge structures by the CFL process. It turns out that $\mathrm{Al}(\mathrm{OH})_{3}$ solidification starts from near the walls and continues in the center of the recessed space of the PDMS stamp used in the CFL process; for example, 20 min of molding time with $1 \mathrm{M} \mathrm{KOH}$ ink guarantees that the highest and widest ring edges of the protruded $\mathrm{Al}(\mathrm{OH})_{3}$ micro-structures can be fabricated by a dot PDSM stamp ( $\phi: 4.5 \mu \mathrm{m}$, gap: $3 \mu \mathrm{m})$. Using the edge-tailored $\mathrm{Al}(\mathrm{OH})_{3}$ micro-structured template, an advantageous PDMS stamp for the ETL process could be fabricated; for example, ring-like and line-shaped PDMS stamps with trenches of $\sim 100 \mathrm{~nm}$ depth around the contact area were replicated from the edge-tailored $\mathrm{Al}(\mathrm{OH})_{3}$ micro-structured template fabricated by dot- and line-array PDMS stamps. As a result, $700 \mathrm{~nm}$-wide $\mathrm{Au}$ bi-line structures (interval: $\sim 5 \mu \mathrm{m}$ ) were fabricated with MHA-SAM patterning and sequential $\mathrm{Au}$ etching by the microscale replicated line-shaped PDMS stamp (width: $9 \mu \mathrm{m}$, gap: $6 \mu \mathrm{m})$. Our demonstration will be a straightforward example of a relatively cheap methodology for fabricating submicron features using microscale PDMS stamp printing combined with Au nanomembrane lithography.

\section{Acknowledgements}

This work was supported by a Research Grant of Pukyong National University (2016 Year).

\section{References}

1 Y. Chen, Microelectron. Eng., 2015, 135, 57-72.
2 Y. Chen, D. Macintyre and S. Thoms, J. Vac. Sci. Technol., B: Microelectron. Nanometer Struct.-Process., Meas., Phenom., 1999, 17, 2507-2511.

3 W. Hu, K. Sarveswaran, M. Lieberman and G. H. Bernstein, J. Vac. Sci. Technol., B: Microelectron. Nanometer Struct.Process., Meas., Phenom., 2004, 22, 1711-1716.

4 Y. Ishii and J. Taniguchi, Microelectron. Eng., 2007, 84, 912-915.

5 J. Taniguchi, K. Koga, Y. Kogo and I. Miyamoto, Microelectron. Eng., 2006, 83, 940-943.

6 S. Y. Chou, P. R. Krauss and P. J. Renstrom, J. Vac. Sci. Technol., B: Microelectron. Nanometer Struct.-Process., Meas., Phenom., 1996, 14, 4129-4133.

7 M. M. Alkaisi and K. Mohamed, in Lithography, ed. M. Wang, InTech, Rijeka, 2010.

8 H. Lan and Y. Ding, in Lithography, ed. M. Wang, InTech, Rijeka, 2010.

9 D. Ye, P.-I. Wang, Z. Ye, Y. Ou, R. Ghoshal, R. Ghoshal and T.-M. Lu, Microelectron. Eng., 2010, 87, 2411-2415.

10 H. Lim, K.-B. Choi, G. Kim, S. Park, J. Ryu and J. Lee, Microelectron. Eng., 2011, 88, 2017-2020.

11 H. Hauser, B. Michl, V. Kübler, S. Schwarzkopf, C. Müller, M. Hermle and B. Bläsi, Energy Procedia, 2011, 8, 648-653.

12 N. Unno and J. Taniguchi, Microelectron. Eng., 2011, 88, 2149-2153.

13 O. Cherniavskaya, A. Adzic, C. Knutson, B. J. Gross, L. Zang, R. Liu and D. M. Adams, Langmuir, 2002, 18, 7029-7034.

14 R. B. A. Sharpe, B. J. F. Titulaer, E. Peeters, D. Burdinski, J. Huskens, H. J. W. Zandvliet, D. N. Reinhoudt and B. Poelsema, Nano Lett., 2006, 6, 1235-1239.

15 S.-H. Lee, H.-N. Kim, R.-K. Kwak and K. Y. Suh, Langmuir, 2009, 25, 12024-12029.

16 K.-Y. Suh, M. C. Park and P. Kim, Adv. Funct. Mater., 2009, 19, 2699-2712.

17 K. Y. Suh, Y. S. Kim and H. H. Lee, Adv. Mater., 2001, 13, 1386-1389.

18 C.-M. Oh, K. H. Park, J.-H. Choi, S. Hwang, H. Noh, Y. M. Yu and J.-W. Jang, Chem. Mater., 2017, 29, 3863-3872.

19 D. Qin, Y. Xia and G. M. Whitesides, Nat. Protoc., 2010, 5, 491-502.

20 D. A. Weinberger, S. Hong, C. A. Mirkin, B. W. Wessels and T. B. Higgins, Adv. Mater., 2000, 12, 1600-1603.

21 H. Zhang, R. Jin and C. A. Mirkin, Nano Lett., 2004, 4, 14931495.

22 X. Chen, G. Zheng, J. I. Cutler, J.-W. Jang and C. A. Mirkin, Small, 2009, 5, 1527-1530.

23 J.-W. Jang, R. G. Sanedrin, A. J. Senesi, Z. Zheng, X. Chen, S. Hwang, L. Huang and C. A. Mirkin, Small, 2009, 5, 1850-1853.

24 D. Belitskus, J. Electrochem. Soc., 1970, 117, 1097-1099.

25 G. K. Schweitzer and L. L. Pesterfield, The Aqueous Chemistry of the Elements, Oxford University Press, New York, 2010.

26 Y. Cai, Z. Zhao, J. Chen, T. Yang and P. S. Cremer, ACS Nano, 2012, 6, 1548-1556. 